\title{
ON THE EDGEWORTH EXPANSION FOR THE SUM OF A FUNCTION OF UNIFORM SPACINGS
}

\author{
R.J.M.M. DOES \\ Department of Medical Informatics and Statistics, University of Limburg, P.O. Box 616, \\ 6200 MD Maastricht, The Netherlands
}

\section{R. HELMERS}

Centre for Mathematics and Computer Science, P.O. Box 4079, 1009 AB Amsterdam, The Netherlands

\author{
C.A.J. KLAASSEN \\ Department of Mathematics, University of Leiden, P.O. Box 9512, 2300 RA Leiden, The \\ Netherlands
}

Received 18 March 1986; revised manuscript received 17 November 1986

Recommended by R. Bhattacharya

\begin{abstract}
An Edgeworth expansion for the sum of a fixed function $g$ of normed uniform spacings is established under a natural moment assumption and an appropriate version of Cramér's condition. This condition is shown to hold under an easily verifiable and mild assumption on the function $g$. This is done by proving Cramér's condition for statistics of the general type $f(X)$ under quite weak assumptions on the random variable $X$ and the function $f: \mathbb{R}^{m} \rightarrow \mathbb{R}^{k}$.
\end{abstract}

AMS Subject Classification: Primary 62E20; Secondary 62G30, 60F05.

Key words and phrases: Edgeworth expansions; Uniform spacings; Cramér's condition.

\section{Introduction}

Let $U_{1}, U_{2}, \ldots$ be a sequence of independent uniform $(0,1)$ random variables. For $n=1,2, \ldots, U_{1: n} \leq U_{2: n} \leq \cdots \leq U_{n: n}$ denote the ordered $U_{1}, U_{2}, \ldots, U_{n}$. Let $U_{0: n}=0$ and $U_{n+1: n}=1$. Uniform spacings are defined by

$$
D_{j n}=U_{j: n}-U_{j-1: n}, \quad j=1,2, \ldots, n+1 .
$$

Let $g:[0, \infty) \rightarrow \mathbb{R}$ be a fixed nonlinear measurable function and define statistics $T_{n}$ by

$$
T_{n}=\sum_{j=1}^{n+1} g\left((n+1) D_{j n}\right), \quad n=1,2, \ldots
$$


Statistics of this form can be used for testing uniformity.

There has been considerable interest into the asymptotic distribution theory for statistics of type (1.2). An excellent survey of first-order limit theory for statistics of the form (1.2) was given by Pyke (1972) according to whom a study of the rate of convergence for sums of functions of uniform spacings is of interest.

We will use the following well-known characterization, which has been applied by Le Cam (1958) in order to prove first-order limit theorems. Let $Y_{j}, j=$ $1,2, \ldots$, be independent exponential random variables with expectation 1 . Let, for $n=1,2, \ldots$,

$$
W_{n}=\sum_{j=1}^{n+1} g\left(Y_{j}\right)
$$

and

Then

$$
S_{n}=\sum_{j=1}^{n+1}\left(Y_{j}-1\right) .
$$

$$
\mathscr{L}\left(T_{n}\right)=\mathscr{L}\left(W_{n} \mid S_{n}=0\right)
$$

i.e. $T_{n}$ has the same distribution as a sum of independent random variables given another sum of independent random variables.

With the aid of (1.5) Does and Klaassen (1984a,b) proved Berry-Esseen bounds of the order $n^{-1 / 2}$ for the normal approximation for statistics based on uniform spacings under natural moment assumptions. In Does and Helmers (1982) Edgeworth expansions were established for statistics of the form (1.2) under a natural moment assumption and an integrability condition on the simultaneous characteristic function of $(Y-1, g(Y))$. In the present paper it is shown that the latter integrability condition can be replaced by a much weaker and more natural Cramér-type condition. This condition holds under an easily verifiable and mild assumption on the function $g$, as is stated in Theorem 2.1. Cramér's condition for statistics of the general type $f(X)$, where $X$ is a random variable taking values in $\mathbb{R}^{\prime \prime}$ and $f: \mathbb{R}^{\prime \prime} \rightarrow \mathbb{R}^{k}$ is a measurable function, is studied in Lemma 3.1.

\section{An Edgeworth expansion}

Let $Y$ be an exponential random variable with expectation 1 and let $g$ be a fixed real-valued measurable function defined on $\mathbb{R}^{+}$. Introduce, whenever well-defined, a function $\tilde{g}$ by

where

$$
\tilde{g}(y)=(g(y)-\mu-\tau(y-1))\left(\sigma^{2}-\tau^{2}\right)^{-1 / 2}, \quad y>0,
$$

and

$$
\mu=E g(Y), \quad \sigma^{2}=\operatorname{Var} g(Y)
$$

$$
\tau=\operatorname{Cov}(g(Y), Y)
$$


Note that $\sigma^{2}=\tau^{2}$ iff $g$ is linear and hence $T_{n}$ is degenerate.

We shall establish an asymptotic expansion with uniform remainder $\mathrm{o}\left(n^{-1}\right)$ for the distribution function

$$
F_{n}(x)=P\left(\left(n\left(\sigma^{2}-\tau^{2}\right)\right)^{-1 / 2}\left(T_{n}-(n+1) \mu\right) \leq x\right), \quad x \in \mathbb{R} .
$$

Let $\varrho$ denote the characteristic function of $(Y-1, g(Y))$, i.e.

$$
\varrho(s, t)=E \mathrm{e}^{\mathrm{i} s(Y-1)+\mathrm{i} / g(Y)}, \quad(s, t) \in \mathbb{R}^{2} .
$$

Let $\Phi$ and $\phi$ denote the distribution function and density of the standard normal distribution and let $\|\cdot\|$ denote the Euclidian norm in $\mathbb{R}^{2}$, i.e. $\|(s, t)\|=\left(s^{2}+t^{2}\right)^{1 / 2}$, for $(s, t) \in \mathbb{R}^{2}$.

Theorem 2.1. Let $F_{n}$ be as in (2.4) (cf. (1.2), (2.2) and (2.3)) and let $g:[0, \infty) \rightarrow \mathbb{R}$ be a measurable function such that

$$
E g^{4}(Y)<\infty
$$

and for an interval $(c, d) \subset(0, \infty)$ on which $g$ is almost everywhere differentiable with derivative $g^{\prime}$,

$$
g^{\prime} \text { is not essentially constant on }(c, d) \text {; }
$$

then

$$
\lim _{n \rightarrow \infty} n \sup _{x \in \mathbb{R}}\left|F_{n}(x)-\tilde{F}_{n}(x)\right|=0,
$$

where

$$
\begin{aligned}
\tilde{F}_{n}(x)=\Phi(x)-\phi(x)\{ & n^{-1 / 2}\left(\frac{1}{6} \kappa_{3}\left(x^{2}-1\right)+a\right)+n^{-1}\left(\frac{1}{24} \kappa_{4}\left(x^{3}-3 x\right)\right. \\
& \left.\left.+\frac{1}{72} \kappa_{3}^{2}\left(x^{5}-10 x^{3}+15 x\right)+\frac{1}{8}\left(-4 a \kappa_{3}+b\right) x+\frac{1}{6} a \kappa_{3} x^{3}\right)\right\}
\end{aligned}
$$

with

$$
\begin{aligned}
& \kappa_{3}=E \tilde{g}^{3}(Y), \quad \kappa_{4}=E \tilde{g}^{4}(Y)-3-3\left\{\left(E \tilde{g}^{2}(Y)(Y-1)\right\}^{2},\right. \\
& a=-\frac{1}{2} E \tilde{g}(Y)(Y-1)^{2}, \\
& b=3\left\{E \tilde{g}(Y)(Y-1)^{2}\right\}^{2}-2 E \tilde{g}^{2}(Y)(Y-1)^{2}+4 E \tilde{g}^{2}(Y)(Y-1)+6 .
\end{aligned}
$$

We note that Condition (2.6) is obviously necessary for the expansion (2.9) to be well-defined.

Instead of Condition (2.7) on the function $g$, Does and Helmers (1982) used an integrability condition on the simultaneous characteristic function of $(Y-1, g(Y))$, i.e.

$$
\int_{-\infty}^{\infty} \int_{-\infty}^{\infty}|\varrho(s, t)|^{p} \mathrm{~d} s \mathrm{~d} t<\infty, \quad \text { for some } p \geq 1,
$$

to validate the expansion (2.9). Integrability conditions like (2.11) are commonly encountered in problems of establishing asymptotic expansions for conditional 
distributions (see e.g. Michel (1979)). An assumption equivalent to (2.11) is that there exists an integer $k$ such that the $k$-th convolution of $(Y-1, g(Y))$ has a bounded density (cf. Bhattacharya and Rao (1976), Theorem 19.1). According to the Riemann-Lebesgue lemma (cf. Theorem 4.1 in Bhattacharya and Rao (1976)) this implies that $|\varrho(s, t)|^{k} \rightarrow 0$ as $\|(s, t)\| \rightarrow \infty$. Consequently (2.11) is much stronger than

$$
\limsup _{\|(s, t)\| \rightarrow \infty}|\varrho(s, t)|<1 .
$$

From the proof of Theorem 2.1 it is clear that (2.6) and Cramér's condition (2.12) suffice for (2.8) to hold. Lemma 3.1 shows that (2.7) implies (2.12). The fact is that this lemma gives conditions for the validity of Cramér's condition for statistics of the general type $f(X)$, where $X$ is a random $m$-vector and $f: \mathbb{R}^{m} \rightarrow \mathbb{R}^{k}$ is a measurable function.

Lemma 3.1 extends Lemma 1.4 of Bhattacharya (1977) with a new and simpler proof. Another way to prove Cramér's condition for a statistic of type $f(X)$ is to show the stronger property that its distribution has a nonzero absolutely continuous component with respect to Lebesgue measure. This problem is treated in Sadikova (1966), Yurinskii (1972) and Bhattacharya and Ghosh (1978). The conditions of Lemma 2.2 of Bhattacharya and Ghosh (1978) are a little bit more restrictive than the conditions of Lemma 3.1, but the conclusion of their Lemma 2.2 is much stronger.

In Section 3 of Pyke (1965) some examples of functions $g$ are given. These functions are related to $g_{1}(x)=x^{r}, \quad r>0, \quad r \neq 1, \quad g_{2}(x)=|x-1|, \quad g_{3}(x)=\log x$ and $g_{4}(x)=x^{-1}$. The functions $g_{1}, g_{2}$ and $g_{3}$ are all included in Theorem 2.1. Note that $g_{4}$ does not satisfy (2.6).

If we standardize the statistic $T_{n}$ (cf. (1.2)) exactly then it should be possible to verify that under the assumptions of Theorem 2.1 relation (2.8) holds, with $F_{n}$ replaced by the distribution function of $\left(T_{n}-E T_{n}\right)\left(\operatorname{Var} T_{n}\right)^{-1 / 2}$ and $\tilde{F}_{n}$ by the righthand side of (2.9) with $a=b=0$. One may prove this by a refinement of Lemma 3.4 from Does and Klaassen (1984a).

We note that, although we have proved our results for a fixed function $g$ it seems to be possible to generalize Theorem 2.1 to functions $g_{j n}$; i.e. functions depending on the $j$-th spacing and sample size $n$. A Berry-Esseen theorem for this more general case was proved in Does and Klaassen (1984b).

\section{Proof of Theorem 2.1}

Without loss of generality we may replace $g$ by $\tilde{g}$ (cf. (2.1)), because this does not affect $F_{n}$ and the assumptions of the theorem. In other words we assume that $\mu=0$, $\sigma^{2}=1$ and $\tau=0$ (cf. (2.2) and (2.3)). Let $\chi_{n}$ denote the characteristic function of $n^{-1 / 2} T_{n}$; i.e. 


$$
\chi_{n}(t)=\int_{-\infty}^{\infty} \mathrm{e}^{\mathrm{i} t x} \mathrm{~d} F_{n}(x),
$$

with $F_{n}$ as in (2.4). By Esseen's smoothing lemma (see e.g. Feller (1971), Lemma $\mathrm{XVI} 3.2$ ) it suffices to prove that

$$
\int_{|t| \leq n \log n} \frac{\left|\chi_{n}(t)-\tilde{\chi}_{n}(t)\right|}{|t|} \mathrm{d} t=\mathrm{o}\left(n^{-1}\right),
$$

where $\tilde{\chi}_{n}$ is the Fourier-Stieltjes transform of $\tilde{F}_{n}$ (cf. (2.9)); i.e.

$$
\begin{aligned}
\tilde{\chi}_{n}(t) & =\int_{-\infty}^{\infty} \mathrm{e}^{\mathrm{i} t x} \mathrm{~d} \tilde{F}_{n}(x) \\
& =\mathrm{e}^{-t^{2} / 2}\left\{1+\frac{a}{n^{1 / 2}} \mathrm{i} t-\frac{\kappa_{3}}{6 n^{1 / 2}} \mathrm{i} t^{3}-\frac{b}{8 n} t^{2}+\frac{\left(\kappa_{4}+4 a \kappa_{3}\right)}{24 n} t^{4}-\frac{\kappa_{3}^{2}}{72 n} t^{6}\right\} .
\end{aligned}
$$

Since

$$
\begin{aligned}
\left|t^{-1}\left(\chi_{n}(t)-1\right)\right| & \leq E\left|n^{-1 / 2} T_{n}\right| \leq n^{-1 / 2}(n+1) E\left|g\left((n+1) D_{1 n}\right)\right| \\
& =\left.n^{1 / 2}\right|_{0} ^{n+1}|g(y)|\left(1-\frac{y}{n+1}\right)^{n-1} \mathrm{~d} y \leq\left.\mathrm{e}^{2} n^{1 / 2}\right|_{0} ^{\infty}|g(y)| \mathrm{e}^{-y} \mathrm{~d} y,
\end{aligned}
$$

for any $t, \chi_{n}(0)=\tilde{\chi}_{n}(0)=1$ and $\tilde{\chi}_{n}$ has a bounded continuous derivative with respect to $t$, it is easily verified (see also (2.50) of Bickel and Van Zwet (1978)) that

$$
\int_{|t| \leq n=2}|t|^{-1}\left|\chi_{n}(t)-\tilde{\chi}_{n}(t)\right| \mathrm{d} t=\mathrm{O}\left(n^{-3 / 2}\right) .
$$

According to Lemma 3.1 of Does and Klaassen (1984a) we can choose a regular version of the conditional distribution of $n^{-1 / 2} W_{n}$ given $n^{-1 / 2} S_{n}=x$ (cf. (1.3)-(1.5)), such that for this version

$$
\chi_{n}(t)=E \mathrm{e}^{\mathrm{i} / n 1 / 2} T_{n}=E\left(\mathrm{e}^{\mathrm{i} t n 1 / 2} W_{n} \mid n^{-1 / 2} S_{n}=0\right)
$$

Let $\psi_{n}$ be the characteristic function of $\left(n^{-1 / 2} S_{n}, n^{-1 / 2} W_{n}\right)$; i.e.

$$
\psi_{n}(s, t)=\left[\varrho\left(s n^{-1 / 2}, t n^{-1 / 2}\right)\right]^{n+1},
$$

with $\varrho$ as in (2.5).

With the aid of Plancherel's identity (see e.g. Theorem 4.1 of Bhattacharya and Rao (1976)) we check that for all $t$ (cf. Does and Klaassen (1984a), formulas (3.15) and (3.16)),

$$
\begin{aligned}
\int_{-\infty}^{\infty}\left|\psi_{n}(s, t)\right| \mathrm{d} s & =\int_{-\infty}^{\infty}\left|\varrho\left(s n^{-1 / 2}, t n^{-1 / 2}\right)\right|^{n+1} \mathrm{~d} s \\
& \leq\left. n^{1 / 2}\right|_{-\infty} ^{\infty}\left|\varrho\left(s, t n^{-1 / 2}\right)\right|^{2} \mathrm{~d} s=\left.2 \pi n^{1 / 2}\right|_{0} ^{\infty} \mathrm{e}^{-2 y} \mathrm{~d} y=\pi n^{1 / 2}
\end{aligned}
$$

Let $h_{n}$ be the density of $n^{-1 / 2} S_{n}$. In view of Lemma 3.1 of Does and Klaassen 
(1984a), (3.5) and (3.7), Fourier inversion of

yields

$$
\psi_{n}(s, t)=\int_{-\infty}^{\infty} \mathrm{e}^{\mathrm{i} s x}\left\{E\left(\mathrm{e}^{\mathrm{i} / n 1^{1} \cdot w_{n}} \mid n^{-1 / 2} S_{n}=x\right) h_{n}(x)\right\} \mathrm{d} x
$$

$$
\chi_{n}(t)=\left(2 \pi h_{n}(0)\right)^{-1} \int_{-\infty}^{\infty} \psi_{n}(s, t) \mathrm{d} s .
$$

An argument like in (3.7) shows that for any $\delta$ and $t$,

$$
\int_{|s| \geq \delta n^{\prime}:}\left|\psi_{n}(s, t)\right| \mathrm{d} s \leq \pi n^{1 / 2} \sup _{|s| \geq \delta}\left|\varrho\left(s, t n^{-1 / 2}\right)\right|^{n-1} .
$$

To show that (3.10) is exponentially small we use the following lemma.

Lemma 3.1. Let $X$ be a random variable taking values in $\mathbb{R}^{\prime \prime}$, the distribution of which is absolutely continuous on some Borel set $B$ with $P(X \in B)>0$. Let $f: \mathbb{R}^{\prime \prime} \rightarrow \mathbb{R}^{k}$ be a measurable function, which is Lebesgue almost everywhere differentiable on $B$ with the $k \times m$ matrix $\dot{f}$ as differential. If all $\gamma \in \mathbb{R}^{k} \backslash\{0\}$ satisfy

then

$$
P\left((\dot{f}(X))^{\mathrm{T}} \gamma=0 \mid X \in B\right)<1,
$$

holds.

$$
\limsup _{\|v\| \rightarrow \infty}\left|E \mathrm{e}^{\mathrm{i} u^{\mathrm{i}} f(x)}\right|<1
$$

Proof. See Appendix.

By taking $m=1, k=2, f(x)=(x, \tilde{g}(x))$ and $B=(c, d)$ in Lemma 3.1 and in view of Condition (2.7) it follows that (2.12) holds for $g$ replaced by $\tilde{g}$ and that hence (3.10) is exponentially small.

The theory of asymptotic expansions for the density of a sum of independent and identically distributed random variables (see e.g. Feller (1971), Theorem XVI 2.2) implies

$$
h_{n}(0)=\frac{1}{(2 \pi)^{1 / 2}}\left(1-\frac{7}{12 n}\right)+\mathrm{O}\left(n^{-3 / 2}\right) .
$$

With the definition (cf. Does and Helmers (1982), formula (3.5))

$$
\begin{aligned}
\tilde{\psi}_{n}(s, t)= & \mathrm{e}^{-\left(s^{2}+t^{2}\right) / 2}\left\{1-\frac{\mathrm{i}}{6 n^{1 / 2}}\left(E \tilde{g}^{3}(Y) t^{3}+3 E \tilde{g}^{2}(Y)(Y-1) t^{2} s\right.\right. \\
& \left.+3 E \tilde{g}(Y)(Y-1)^{2} t s^{2}+2 s^{3}\right)+\frac{1}{24 n}\left(\left(E \tilde{g}^{4}(Y)-3\right) t^{4}\right. \\
& +4 E \tilde{g}^{3}(Y)(Y-1) t^{3} s+6\left\{E \tilde{g}^{2}(Y)(Y-1)^{2}-1\right\} t^{2} s^{2} \\
& \left.+4 E \tilde{g}(Y)(Y-1)^{3} t s^{3}+6 s^{4}\right)
\end{aligned}
$$




$$
\begin{aligned}
& -\frac{1}{72 n}\left(E \tilde{g}^{3}(Y) t^{3}+3 E \tilde{g}^{2}(Y)(Y-1) t^{2} s\right. \\
& \left.\left.+3 E \tilde{g}(Y)(Y-1)^{2} t s^{2}+2 s^{3}\right)^{2}-\frac{1}{2 n}\left(t^{2}+s^{2}\right)\right\}
\end{aligned}
$$

and (3.13), we find after some computations (cf. (3.3))

$$
\tilde{\chi}_{n}(t)=\left(2 \pi h_{n}(0)\right)^{-1} \int_{-\infty}^{\infty} \tilde{\psi}_{n}(s, t) \mathrm{d} s+\mathrm{O}\left(n^{-3 / 2}\left(1+t^{6}\right) \mathrm{e}^{-t^{2} / 2}\right),
$$

uniformly in $t$.

Hence, in view of (3.2), (3.4), (3.9), the exponential bound on (3.10), (3.13), (3.14) and (3.15), it suffices to show that for some $\delta>0$,

$$
\int_{n^{2} \leq|t| \leq n \log n}|t|^{-1}\left|\int_{|s| \leq \delta n^{12}}\left\{\psi_{n}(s, t)-\tilde{\psi}_{n}(s, t)\right\} \mathrm{d} s\right| \mathrm{d} t=\mathrm{o}\left(n^{-1}\right) .
$$

Application of Theorem 9.12 of Bhattacharya and Rao (1976) (with $V=I, s=4$, $k=2$ and $\alpha=(0,0))$ yields

$$
\int_{1 \leq|t| \leq \epsilon n^{1}=}|t|^{-1} \int_{|s| \leq \delta n^{\prime}=}\left|\psi_{n}(s, t)-\tilde{\psi}_{n}(s, t)\right| \mathrm{d} s \mathrm{~d} t=\mathrm{o}\left(n^{-1}\right),
$$

for some $\varepsilon>0$ and $\delta>0$. By the same theorem (with $\alpha=(0,1)$ ) we obtain

$$
\int_{n^{2} \leq|t| \leq 1} \mid \int_{|s| \leq \delta n^{\prime}=2} \frac{1}{t}\left\lceil\frac{\partial}{\partial u}\left[\psi_{n}(s, u)-\tilde{\psi}_{n}(s, u)\right] \mathrm{d} u \mathrm{~d} s \mid \mathrm{d} t=\mathrm{o}\left(n^{-1}\right) .\right.
$$

Moreover, the classical theory of Edgeworth expansions for sums of independent and identically distributed random variables yields

$$
\int_{n^{2} \leq|t| \leq 1}\left|\frac{1}{t} \int_{|s| \leq \delta n^{1 / 2}}\left\{\psi_{n}(s, 0)-\tilde{\psi}_{n}(s, 0)\right\} \mathrm{d} s\right| \mathrm{d} t=\mathrm{O}\left(n^{-3 / 2} \log n\right) .
$$

Combining (3.16)-(3.19) we see that it remains to show that

$$
\int_{\varepsilon n^{1} \leq \leq|t| \leq n \log n} \int_{|s| \leq \delta n^{\prime}:}\left\{\left|\frac{\psi_{n}(s, t)}{t}\right|+\left|\frac{\tilde{\psi}_{n}(s, t)}{t}\right|\right\} \mathrm{d} s \mathrm{~d} t=\mathrm{o}\left(n^{-1}\right) \text {. }
$$

But this is easily seen to be a simple consequence of formula (3.14), Lemma 3.1 and Condition (2.7) (cf. the argument after Lemma 3.1). This completes the proof of Theorem 2.1.

Remark 3.1. As in the classical proof of Edgeworth expansions for sums of independent and identically distributed random variables, one needs a condition to guarantee that for all $\varepsilon>0$,

$$
\int_{\varepsilon n^{12} \leq|t| \leq n \log n}\left|\frac{\chi_{n}(t)}{t}\right| \mathrm{d} t=\mathrm{o}\left(n^{-1}\right)
$$

In view of (3.9), (3.13), (3.6) and an argument like (3.10), condition (3.21) is implied 
by and presumably almost equivalent to

$$
\int_{\varepsilon n^{1} \geq \leq|t| \leq n \log n}|t|^{-1} \sup _{s \in \mathbb{K}^{\mathbb{R}}}\left|\varrho\left(s n^{-1 / 2}, t n^{-1 / 2}\right)\right|^{n-1} \mathrm{~d} t=\mathrm{o}\left(n^{-3 / 2}\right) .
$$

The natural Cramér-type condition to ensure this reads as follows:

$$
\sup _{|t| \geq \varepsilon} \sup _{s \in \mathbb{R}}|\varrho(s, t)|<1, \quad \text { for any } \varepsilon>0 .
$$

Under (2.6) this is easily checked to be equivalent to (2.12).

\section{Appendix}

Without loss of generality we assume $B=\mathbb{R}^{m}$. Let $\left\{v_{j}\right\}$ be a sequence in $\mathbb{R}^{k}$ with $\left\|v_{j}\right\| \rightarrow \infty$ and

$$
\lim _{j \rightarrow \infty}\left|E \mathrm{e}^{\mathrm{i} \omega_{j}^{1} f(X)}\right|=\limsup _{\|u\| \rightarrow \infty}\left|E \mathrm{e}^{\mathrm{i} \omega^{1} f(X)}\right| .
$$

Let $w_{j}$ be the maximum of the absolute values of the coordinates of $v_{j}$ and assume, by selecting subsequences if necessary, that $w_{j}^{-1} v_{j}$ converges to some $\gamma \in \mathbb{R}^{k} \backslash\{0\}$. Let $z \in \mathbb{R}^{\prime \prime}$ and define $\varepsilon_{j}=w_{j}^{-1} z$. Then $\varepsilon_{j} \rightarrow 0$, as $j \rightarrow \infty$, and since $X$ has a density on $\mathbb{R}^{\prime \prime}$, the $\mathscr{L}_{1}\left(\mathbb{R}^{m}\right)$-continuity of translations implies

$$
E \mathrm{e}^{\mathrm{i} \omega_{j}^{!} f(X)}=E \mathrm{e}^{\mathrm{i} \omega_{j}^{1} f\left(X+\varepsilon_{j}\right)}+\mathrm{o}(1), \quad \text { as } j \rightarrow \infty .
$$

Because $f$ is almost everywhere differentiable this yields

$$
E \mathrm{e}^{\mathrm{i} \nu^{\prime}, f(X)}=E \mathrm{e}^{\mathrm{i} \nu_{l}^{\mathrm{l}} f(X)+\mathrm{i} \gamma^{\prime} f(x) \approx}+\mathrm{o}(1) .
$$

With $Z$ a N $\left(0, I_{m}\right)$ random variable, $I_{m}$ the $m \times m$ identity matrix, $X$ and $Z$ independent, we obtain from (A.3),

$$
\begin{aligned}
& \lim _{j \rightarrow \infty}\left|E \mathrm{e}^{\mathrm{i} \omega_{j}^{\mathrm{T}} f(X)}\right|=\lim _{j \rightarrow \infty}\left|E \mathrm{e}^{\mathrm{i} \nu_{j}^{l} f(X)+\mathrm{i} \gamma^{\prime} f(X) Z}\right| \\
& =\lim _{j \rightarrow \infty}\left|E \mathrm{e}^{\mathrm{i} \omega_{j}^{\top} f(X)} \mathrm{e}^{-\left\|(f(X))^{1} y\right\|^{2} / 2}\right| \\
& \leq E \mathrm{e}^{-\left\|(\dot{f}(X))^{1} \gamma\right\|^{2} / 2} .
\end{aligned}
$$

Combining (A.1) and (A.4) we see that (3.12) can be violated only if there exists a $\gamma \in \mathbb{R}^{k} \backslash\{0\}$ such that $(\dot{f}(X))^{\mathrm{T}} \gamma$ is degenerate at 0 . In view of (3.11) this can not be the case.

\section{Acknowledgements}

The authors are very grateful to the editor and the associate editor for their comments and suggestions. 


\section{References}

Bhattacharya, R.N. (1977). Refinements of the multidimensional limit theorem and applications. Ann. Probab. 5, 1-27.

Bhattacharya, R.N. and J.K. Ghosh (1978). On the validity of the formal Edgeworth expansion. Ann. Statist. 6, 434-451.

Bhattacharya, R.N. and R.R. Rao (1976). Normal Approximation and Asymptotic Expansions. Wiley, New York.

Bickel, P.J. and W.R. van Zwet (1978). Asymptotic expansions for the power of distribution free tests in the two-sample problem. Ann. Statist. 6, 937-1004.

Does, R.J.M.M. and R. Helmers (1982). Edgeworth expansions for functions of uniform spacings. In: B.V. Gnedenko, M.L. Puri and I. Vincze, Eds., Nonparametric Statistical Inference, Coll. Math. Soc. J. Bolyai, Vol. 32. North-Holland, Amsterdam, 203-212.

Does, R.J.M.M. and C.A.J. Klaassen (1984a). The Berry-Esseen theorem for functions of uniform spacings. Z. Wahrsch. Verw. Geb. 65, 461-471.

Does, R.J.M.M. and C.A.J. Klaassen (1984b). Second order asymptotics for statistics based on uniform spacings. In: P. Mandl and M. Hušková, Eds., Asymptotic Statistics 2. North-Holland, Amsterdam, 231-239.

Feller, W. (1971). An Introduction to Probability Theory and Its Applications, Vol. II, 2nd edition. Wiley, New York.

Le Cam, L. (1958). Un théorème sur la division d'un intervalle par des points pris au hasard. Publ. Inst. Statist. Univ. Paris 7, 7-16.

Michel, R. (1979). Asymptotic expansions for conditional distributions. J. Multivariate Anal. 9, 393-400.

Pyke, R. (1965). Spacings. J. Roy. Statist. Soc. Ser. B 27, 395-449.

Pyke, R. (1972). Spacings revisited. Proc. Sixth Berkeley Sympos. Math. Statist. Probability 1, 417-427.

Sadikova, S.M. (1966). Some inequalities for characteristic functions. Theory Probab. Appl. 11, 441-447.

Yurinskii, V.V. (1972). Bounds for characteristic functions of certain degenerate multidimensional distributions. Theory Probab. Appl. 17, 101-113. 